\title{
Converging Evidence for the Neuroanatomic Basis of Combinatorial Semantics in the Angular Gyrus
}

\author{
Amy R. Price, ${ }^{1,2}$ Michael F. Bonner, ${ }^{1}$ Jonathan E. Peelle, ${ }^{3}$ and Murray Grossman ${ }^{1}$ \\ ${ }^{1}$ Penn FTD Center and Department of Neurology and ${ }^{2}$ Neuroscience Graduate Group, University of Pennsylvania, Philadelphia, Pennsylvania 19104 and \\ ${ }^{3}$ Department of Otolaryngology, Washington University in St. Louis, St. Louis, Missouri 63130
}

\begin{abstract}
Human thought and language rely on the brain's ability to combine conceptual information. This fundamental process supports the construction of complex concepts from basic constituents. For example, both "jacket" and "plaid" can be represented as individual concepts, but they can also be integrated to form the more complex representation "plaid jacket." Although this process is central to the expression and comprehension of language, little is known about its neural basis. Here we present evidence for a neuroanatomic model of conceptual combination from three experiments. We predicted that the highly integrative region of heteromodal association cortex in the angular gyrus would be critical for conceptual combination, given its anatomic connectivity and its strong association with semantic memory in functional neuroimaging studies. Consistent with this hypothesis, we found that the process of combining concepts to form meaningful representations specifically modulates neural activity in the angular gyrus of healthy adults, independent of the modality of the semantic content integrated. We also found that individual differences in the structure of the angular gyrus in healthy adults are related to variability in behavioral performance on the conceptual combination task. Finally, in a group of patients with neurodegenerative disease, we found that the degree of atrophy in the angular gyrus is specifically related to impaired performance on combinatorial processing. These converging anatomic findings are consistent with a critical role for the angular gyrus in conceptual combination.
\end{abstract}

Key words: angular gyrus; combinatorial semantics; conceptual combination; compositionality; semantic integration; semantic memory

\section{Introduction}

A major goal of neuroscience is to understand the neural basis of behaviors that are fundamental to human intelligence. One such behavior is the ability to combine conceptual information in language and thought. This combinatorial process allows humans to dynamically construct an unlimited number of complex concepts from a finite set of constituents. For example, we can take the basic concepts "leaf" and "wet" and combine them to create the representation of a "wet leaf." The cognitive processes supporting conceptual combination have long been investigated in psychology and philosophy, but little is known about their neural basis (Hume, 1739; Fodor and LePore, 2002; Murphy, 2002).

Most neuroanatomic theories of semantic memory have focused on the representation of individual concepts (Pulvermüller, 2005; Martin, 2007; Patterson et al., 2007; Binder et al., 2009). Much of this work has examined the role of sensory and motor association cortices in representing the features of individual

\footnotetext{
Received Aug. 18, 2014; revised Dec. 19, 2014; accepted Jan. 8, 2015.

Author contributions: A.R.P., M.F.B., J.E.P., and M.G. designed research; A.R.P. performed research; A.R.P. analyzed data; A.R.P., M.F.B., J.E.P., and M.G. wrote the paper.

This work was supported by the National Institutes of Health (AG017586, AG032953, AG038490, NS044266, NS053488, and AG00255), the Wyncote Foundation, and travel award support from Fine Science Tools. We are grateful to all of the patients and the caregivers for their participation in this study.

The authors declare no competing financial interests.

Correspondence should be addressed to Amy R. Price, Department of Neurology-2 Gibson, University of Pennsylvania, 3400 Spruce Street, Philadelphia, PA 19104. E-mail: amyprice@mail.med.upenn.edu.

DOI:10.1523/JNEUROSCI.3446-14.2015

Copyright $\odot 2015$ the authors $\quad 0270-6474 / 15 / 353276-09 \$ 15.00 / 0$
}

concepts (e.g., the sound feature of "thunder" is thought to be represented in or near auditory association cortex; Bonner and Grossman, 2012). Some of this work has also examined the role of high-level heteromodal association cortices, sometimes referred to as "hubs," in representing the amodal associations of concepts (e.g., the intrinsic knowledge that "apples" are edible fruit; Patterson et al., 2007; Binder et al., 2009). However, few studies have directly examined the neural basis for how individual concepts are combined into more complex representations.

Here we test the prediction that conceptual combination relies in part on the heteromodal association cortex of the angular gyrus. Anatomically, the angular gyrus is well situated to perform this type of integration. It has widespread white matter connectivity with sensory and motor association cortices as well as with classic language regions, such as the inferior frontal and superior temporal cortices (Seltzer and Pandya, 1978; Pandya and Seltzer, 1982; Yeterian and Pandya, 1985; Mesulam and Mesulam, 2000; Bonner et al., 2013). The cytoarchitectonic properties of the angular gyrus also reflect a specialization for high-level multimodal processing: Relative to unimodal cortices, heteromodal brain regions like the angular gyrus have larger and more complex dendritic fields, indicating diverse and highly integrative computations (Elston et al., 2001; Jacobs et al., 2001). Furthermore, the angular gyrus is one of the most commonly activated regions in functional neuroimaging studies of semantic memory (Binder et al., 2009).

Here we demonstrate that the angular gyrus supports the integration of individual concepts into coherent semantic combi- 
nations. In three experiments we find that: (1) neural activity in the angular gyrus increases during conceptual combination, (2) anatomic variability in the angular gyrus in healthy adults predicts individual differences in the processing of combined concepts, and (3) atrophy of the angular gyrus in patients with neurodegenerative disease results in impaired conceptual combination. These findings build on previous work that more broadly implicates the angular gyrus in semantic representation, and indicate a specific, high-level function for semantic integration.

\section{Materials and Methods}

\section{Healthy adult experiments}

Participants. Twenty-two healthy adults from the University of Pennsylvania community participated in the study ( 10 females; mean age $=25.3$ years; range $=19-36$ ). All were right-handed, native English speakers with no history of neurological difficulty, as determined by a preexperiment screening. We obtained informed consent from all participants according to a protocol approved by the University of Pennsylvania Institutional Review Board.

Experimental design and task stimuli. Our experimental design aimed to isolate the neural activity associated with the basic process of combining conceptual information in a semantically meaningful manner. To do this, we examined the processing of meaningful adjective-noun combinations. We created sets of word pairs that systematically varied in how readily the words could be integrated into a combined concept (as determined in a series of norming studies, discussed below). The word pairs could be divided into pairs that readily combined to form meaningful conceptual combinations (e.g., plaid jacket) and pairs that did not readily combine to form meaningful combinations (e.g., moss pony). We also manipulated the type of sensorimotor information associated with the combination so that there were four different sensorimotor semantic categories of meaningful combinations: auditory, motion, tactile, and visual. For example, the first word modified the second word in a manner that was strongly auditory $(n=28$; e.g., loud car), motion $(n=28$; e.g., drifting balloon), tactile ( $n=28$; e.g., gooey candy), or visual $(n=28$; e.g., plaid jacket). The second word always referred to a concrete object. We refer to these four categories here as the "meaningful combinatorial word pairs."

To develop the stimuli, we first collected association ratings from 20 young adults on 371 adjectives and 489 nouns for how strongly each word was associated with each of the four sensorimotor features on a 1 to 7 scale: sound, motion, tactile, and visual association ratings. From these, we then created four categories of word pairs ( $n=28$ per category) based on the sensory-association ratings of the modifier (i.e., the first word). These word pairs were balanced on summed values for letter length, word frequency (Brysbaert and New, 2009), co-occurrence frequency (see below), orthographic neighborhood density (Medler and Binder, 2005), and syllable number (all pairwise $t$ test comparisons, $p>0.2$ ). Across all conditions, nouns were highly imageable and did not differ on any of the four sensorimotor feature associations (all pairwise $t$ test comparisons, $p>0.2$ ). We also collected sensorimotor feature association ratings on a 1 to 7 scale for each word pair (i.e., at the phrase level) to confirm that the phrase-level associations were similar to those obtained in the singleword norming data. Sound feature associations were highest for the sound word pairs [sound association ratings: sound word pairs $=6.5$ $(0.4)$; motion word pairs $=2.5(0.8)$; tactile word pairs $=1.5(0.5)$; visual word pairs $=1.3(0.2)$; all $t$ tests $p<0.001$ in comparisons of sound words with other groups]. Motion feature associations were highest for the motion word pairs [motion association ratings: motion word pairs $=$ $6.2(0.6)$; sound word pairs $=3.1(0.6)$; tactile word pairs $=1.5(0.3)$; visual word pairs $=1.4(0.4)$; all $t$ tests $p<0.001$ in comparisons of motion words with other groups]. Tactile feature associations were highest for the tactile word pairs [tactile association ratings: tactile word pairs $=5.8(0.6)$; sound word pairs $=1.6(0.6)$; motion word pairs $=1.5$ $(0.2)$; visual word pairs $=1.6(0.6)$; all $t$ tests $p<0.001$ in comparisons of tactile words with other groups]. Visual feature associations were highest for the visual word pairs [visual association ratings: visual word pairs $=$ $6.7(0.3)$; sound word pairs $=4.2(0.6)$; motion word pairs $=5.4(0.4)$; tactile word pairs $=4.4(0.8)$; all $t$ tests $p<0.001$ in comparisons of visual words with other groups].

We next constructed a baseline of two real words that were judged to combine less meaningfully, which we refer to as the "nonmeaningful" baseline (e.g., moss pony; $n=28$ ). These word pairs did not differ statistically from the meaningful combinatorial word pairs on any of the sum sensorimotor feature associations (auditory, motion, tactile, or visual). They also did not differ statistically from any of the meaningful combinatorial word pair categories on summed values for word frequency (Brysbaert and New, 2009), letter length, concreteness, orthographic neighborhood density, or number of syllables (all pairwise $t$ test comparisons $p>0.1$ ). We also included two low-level baseline conditions containing a pronounceable pseudoword paired with a concrete noun (e.g., sloke road; $n=28$ ) and a pronounceable pseudoword paired with another pronounceable pseudoword (e.g., micked yark; $n=28$ ). These last two were included as additional low-level baselines, but are not used in the analyses presented here.

Next, we collected plausibility ratings on a 1 to 7 scale for all of the word pairs to ensure that subjects considered (1) that meaningful combinatorial word pairs formed highly plausible combinations and (2) that nonmeaningful combinatorial word pairs formed highly implausible combinations ( $n=24$ healthy adults; meaningful combinatorial word pairs average rating $=6.22$; noncombinatorial word pairs average rating $\left.=1.75 ; t_{(138)}=27.5, p<0.001\right)$.

The norming study also revealed that subjects treated the plausibility of conceptual combinations as a continuous factor rather than a dichotomous one (i.e., within the category of meaningful combinations, some concepts formed stronger combinations than others). To explore this issue further, we obtained an objective, quantitative measure of how frequently our stimulus items co-occurred in written text, allowing us to test more fine-grained predictions about the neural basis for conceptual combination. We hypothesized that more frequent word combinations would be judged as more plausible. A large corpus was needed to capture the variability of co-occurrence frequencies across word pairs. To do this we determined the number of times the two words occurred together in a particular order within all web pages ending in ".com" that are indexed on Google (i.e., assessing unidirectional co-occurrence, which is how frequently the words plaid jacket occur together in sequence but not in the reverse sequence "jacket plaid"). Specifically, we identified the number of search hits for a particular word combination and took the log of this value to generate the log co-occurrence frequency. We refer to this measure as the "combinatorial strength" of the word pairs. This measure strongly correlated with the behavioral plausibility ratings of the word pairs that we collected separately in 24 healthy adults (Spearman's $\rho=$ $0.55 ; p<0.001)$. There were no differences in the log co-occurrence frequencies between the four sensorimotor combinatorial categories. The distribution of $\log$ co-occurrence frequencies for all meaningful combinations is illustrated in Figure $2 A$.

Procedure. The fMRI experiment used an event-related design, illustrated schematically in Figure 1. On each trial, a fixation cross was presented for $500 \mathrm{~ms}$ followed by the display of the word pair for $2500 \mathrm{~ms}$. Before the experiment, participants received a practice session to ensure that they understood the task. Participants were instructed to press one button if the displayed word pair formed a meaningful combination and another button if it did not (buttons were randomized equally across participants). Stimuli were presented in a random sequence order across three different scanning blocks with a brief pause between blocks. Onequarter of all events were null events ( $3 \mathrm{~s}$ in duration).

Image acquisition and analysis in healthy adults. Subjects were scanned on a Siemens 3.0 T Trio scanner. We acquired T1-weighted structural images using an MPRAGE protocol $(\mathrm{TR}=1620 \mathrm{~ms}$, TE $=3.9 \mathrm{~ms}$, flip angle $=15^{\circ}, 1 \mathrm{~mm}$ slice thickness, $192 \times 256$ matrix, resolution $=$ $0.9766 \times 0.9766 \times 1 \mathrm{~mm})$, followed by BOLD fMRI images $(\mathrm{TR}=3 \mathrm{~s}$, TE eff $=30 \mathrm{~ms}$, flip angle $=90^{\circ}, 64 \times 64$ matrix, $3 \mathrm{~mm}$ isotropic voxels, and fat saturation)

We processed the T1-weighted structural images with PipeDream (https://sourceforge.net/projects/neuropipedream/) and Advanced Normalization Tools (ANTS; http://www.picsl.upenn.edu/ANTS/; Avants et al., 2008). The images were inhomogeneity corrected using the N4ITK algo- 
rithm (Tustison et al., 2010), warped to a local template space using symmetric diffeomorphic normalization in ANTS, segmented into tissue probability maps using template-based priors, and then registered to MNI-template space. We examined voxel-based cortical thickness using a registration-based cortical thickness measure (Das et al., 2009; Tustison et al., 2014). This method uses a continuous one-to-one correspondence between the gray matter-white matter interface and the gray matter-CSF interface given by a diffeomorphic mapping in the image space, and defines thickness in terms of a distance measure between the interfaces of this sheet-like structure. The preprocessed images were further analyzed using SPM8 (Wellcome Trust Centre for Neuroimaging, London, UK), including smoothing with an $8 \mathrm{~mm}$ FWHM Gaussian kernel.

We processed and analyzed the fMRI images using SPM8. Each subject was modeled individually. All functional images were realigned to the first image, coregistered with the structural image, and normalized to standard MNI space using unified segmentation with resampling of images into isotropic $2 \mathrm{~mm}$ voxels. We inspected movement parameters generated during image realignment. Three participants who moved $>2$ $\mathrm{mm}$ during the functional sessions were excluded from the functional analyses. No other subjects moved $>1 \mathrm{~mm}$ during the entire scan. We spatially smoothed the images using an $8 \mathrm{~mm}$ FWHM isotropic Gaussian kernel. To remove low-frequency drifts, we applied a high-pass filter with a cutoff period of $90 \mathrm{~s}$. We modeled autocorrelations with a first-order autoregressive model. For all whole-brain analyses, we used a clusterdefining threshold of $p<0.001$ uncorrected, and performed a clusterlevel correction for FWE across the whole brain at $p<0.05$ using random field theory (Worsley et al., 1992).

Functional imaging in healthy adults: subject analysis. In the subjectlevel analysis, first-level models were created for each subject individually using a general linear model of the BOLD signal with regressors for each category and with movement parameters included as covariates of no interest. To make inferences across participants, we entered the parameter estimates into a second-level random-effects analysis, with subjects as random variables.

Functional imaging in healthy adults: item-analysis. We performed an item analysis (Bedny et al., 2007) in which each meaningful combinatorial word pair was modeled individually and then averaged across all subjects. The first-level analysis included a covariate for every single meaningful combinatorial item individually $(n=112 ; n=28$ per sensorimotor category), and a single covariate for each of the noncombinatorial conditions plus an additional covariate for each scanning session (yielding a total of $118 \beta$-maps). Next the $\beta$-maps for each covariate were averaged across all subjects to perform the second-level analysis. Thus, a voxel within each $\beta$-map contained the averaged $\beta$-value for that item, derived from all subjects. To test the correlation of combinatorial strength and activity across the 112 combinatorial items, we performed a regression analysis using the combinatorial strength and the individual $\beta$-maps for each item, allowing us to look at the regions that positively correlated with combinatorial strength.

Anatomically defined regions of interest in the healthy adults. ROIs for the left angular gyrus, left middle temporal pole, and right angular gyrus were created using anatomic labels (Tzourio-Mazoyer et al., 2002) defined in the WFU Pick Atlas (Maldjian et al., 2003). We ensured that signal strength in the temporal pole ROI was maximized by constraining it to voxels that had a signal of at least $80 \%$ of the global signal for each participant (Devlin et al., 2000).

\section{Patient experiment}

Participants. To ensure that we would be able to detect possible anatomic effects across a range of cortical regions, we examined a heterogeneous group of patients with cortical atrophy affecting portions of the parietal, frontal, occipital, and temporal lobes. Twenty patients with clinically diagnosed dementia from neurodegenerative disease participated in this study (see Table 1 for details; 7 females). Patients were diagnosed according to published criteria (Albert et al., 2011; Gorno-Tempini et al., 2011; McKhann et al., 2011; Rascovsky et al., 2011) and diagnoses were confirmed in a consensus conference based on a review of a semistructured history, a comprehensive mental status exam, and a complete neurological exam by at least two independent, trained reviewers. All participants
Table 1. Demographic characteristics and behavioral performance of patients

\begin{tabular}{|c|c|c|c|c|c|c|}
\hline Syndrome & $\begin{array}{l}\text { Single-word } \\
\text { task } \\
\text { (accuracy\%) }\end{array}$ & $\begin{array}{l}\text { Combinatorial } \\
\text { task (accuracy } \\
\% \text { ) }\end{array}$ & $\begin{array}{l}\text { Difference } \\
\text { score }(\%)\end{array}$ & $\begin{array}{l}\text { Gray matter density } \\
\text { in angular gyrus }\end{array}$ & $\begin{array}{l}\text { MMSE } \\
\text { score }\end{array}$ & $\begin{array}{l}\text { Age at } \\
\text { test }\end{array}$ \\
\hline$P C A$ & 97.1 & 77.2 & 19.9 & 0.56 & 25 & 59 \\
\hline IvPPA & 91.8 & 73.2 & 18.6 & 0.6 & 17 & 64 \\
\hline bvFTD & 93.9 & 75.4 & 18.5 & 0.56 & 23 & 65 \\
\hline bvFTD & 93.2 & 75.4 & 17.8 & 0.59 & 25 & 61 \\
\hline CBS & 87.7 & 71 & 16.7 & 0.52 & 17 & 62 \\
\hline sVPPA & 80 & 63.8 & 16.2 & 0.59 & 24 & 66 \\
\hline bvFTD & 97.9 & 83.5 & 14.4 & 0.54 & 24 & 63 \\
\hline$P C A$ & 97.1 & 83.9 & 13.2 & 0.56 & 22 & 70 \\
\hline sVPPA & 67.1 & 54.9 & 12.2 & 0.6 & 16 & 64 \\
\hline bvFTD & 96.4 & 88.4 & 8 & 0.6 & 26 & 77 \\
\hline $\mathrm{MCl}$ & 98.9 & 91.1 & 7.9 & 0.63 & 28 & 63 \\
\hline $\mathrm{MCl}$ & 84.6 & 77.2 & 7.4 & 0.59 & 26 & 55 \\
\hline SVPPA & 82.1 & 76.8 & 5.4 & 0.62 & 27 & 70 \\
\hline bvFTD & 97.5 & 92.9 & 4.6 & 0.63 & 28 & 64 \\
\hline IvPPA & 98.2 & 94.2 & 4 & 0.64 & 30 & 72 \\
\hline ALS & 100 & 96 & 4 & 0.65 & 17 & 53 \\
\hline IvPPA & 98.9 & 97.3 & 1.6 & 0.66 & 29 & 52 \\
\hline IvPPA & 96.8 & 95.5 & 1.3 & 0.6 & 29 & 62 \\
\hline IVPPA & 98.9 & 97.8 & 1.2 & 0.62 & 30 & 61 \\
\hline $\mathrm{MCl}$ & 95.7 & 94.6 & 1 & 0.63 & 26 & 66 \\
\hline
\end{tabular}

CBS, corticobasal syndrome; bvFTD, behavioral variant frontotemporal dementia; PCA, posterior cortical atrophy; $\mathrm{MCl}$, mild cognitive impairment; IVPPA, logopenic variant of primary progressive aphasia; SVPPA, semantic variant of primary progressive aphasia; difference score: single-word task accuracy - combinatorial task accuracy; MMSE, mini-mental state examination $(\max =30)$.

and their legal representatives participated in an informed consent procedure approved by the University of Pennsylvania institutional review board.

Experimental design. To allow for direct comparisons with the fMRI study in healthy adults, patients performed the same combinatorial task described above, as well as a previously published single-word lexical decision task (Bonner and Grossman, 2012; Bonner et al., 2013). In the single-word lexical decision, patients viewed a single word presented on the screen and indicated by button press whether the word was a real word (e.g., pyramid) or a pronounceable pseudoword (e.g., dranby). There were no words repeated between the combinatorial and singleword tasks. The words from the combinatorial task and the single-word lexical decision task were additionally matched on average frequency $\left(t_{(270)}=0.05, p=0.96\right)$ and average letter length per word $\left(t_{(270)}=1.03\right.$, $p=0.30)$.

Image acquisition and analysis in patients. T1-weighted structural images were acquired with the same protocol described in the healthy-adult experiment above, and the images were analyzed with PipeDream, ANTS, and SPM8. Gray matter density images were obtained from tissue probability maps through segmentation with template-based priors. These were then registered to MNI-template space and smoothed with an $8 \mathrm{~mm}$ FWHM Gaussian kernel. We constrained all statistical analyses to gray matter using an explicit mask, defined by generating a mean gray matter probability image from all subjects and thresholding at 0.2 . For the whole-brain analysis, we applied the same statistical threshold as in the above analyses in healthy adults, using random field theory with an adjustment for nonstationarity (Hayasaka et al., 2004).

\section{Results}

\section{Behavioral results in healthy adults}

Subjects were highly accurate on the combinatorial semantic judgment task (mean accuracy $=95.5 \%, \mathrm{SD}=3.5$ ). There was no significant difference in accuracy between the meaningful and nonmeaningful combinatorial word-pair conditions $\left(t_{(21)}=1.3\right.$, $p=0.22$ ). Reaction times were faster for the meaningful combinations (meaningful combinatorial mean $=1178.7 \mathrm{~ms}, \mathrm{SD}=$ $215.4 \mathrm{~ms}$; nonmeaningful combinatorial mean $=1358.7 \mathrm{~ms}$, $\left.\mathrm{SD}=231.6 \mathrm{~ms} ; t_{(21)}=5.6, p<0.001\right)$. 
A Task

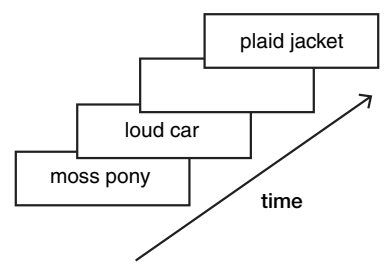

B Meaningful combinations > non-meaningful combinations

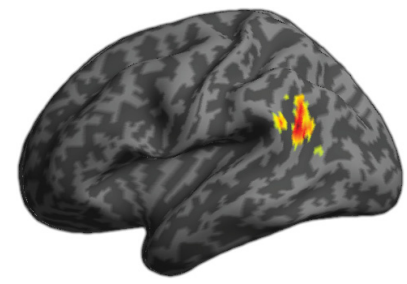

C Individual sensorimotor categories

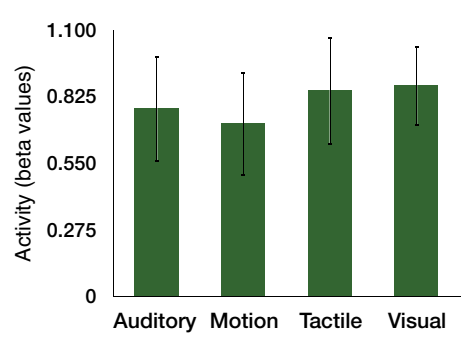

Figure 1. Activity in the angular gyrus was modulated by meaningful combinations, independent of the category of semantic information forming the combination. $A$, Subjects viewed pairs of real words whose combinations were considered to be meaningful (e.g., plaid jacket) or nonmeaningful (e.g., moss pony) based on the results of a norming study. $\boldsymbol{B}$, fMRl activation in healthy adults for the meaningful combinatorial word pairs relative to the nonmeaningful combinatorial word pairs $(p<0.001$ voxelwise, cluster-level $p<0.05$ whole-brain corrected for family wise error; this was a cluster of $4584 \mu \mathrm{l}$ with a peak at [ $-52-5622])$. C, There were no differences in activation between the four sensorimotor semantic categories of meaningful combinations within the left angular gyrus activation cluster shown in $\boldsymbol{B}$ (error bars represent \pm 1 SEM).

A Distribution of combinatorial strength values

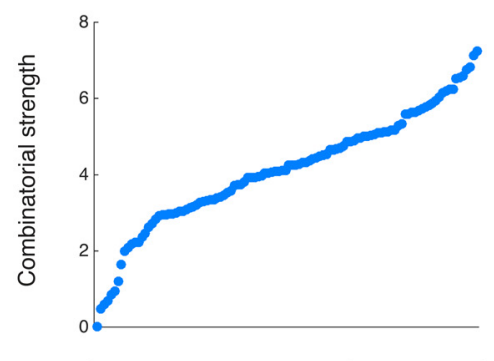

Combinatorial word pairs (ranked order)

B Activity correlates with combinatorial strength
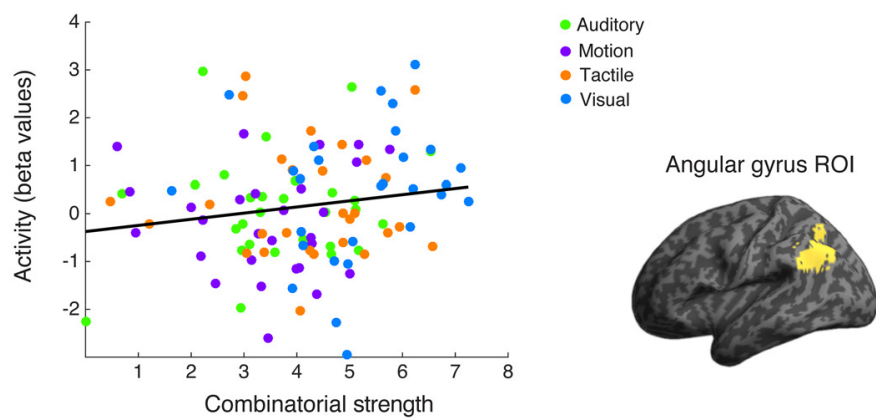

Figure 2. Activity in the angular gyrus increased as a function of the combinatorial strength of individual word pairs modeled in an item analysis. $\boldsymbol{A}$, The distribution of combinatorial strength values (log co-occurrence frequencies) for all meaningful combinatorial word pairs, ranked from lowest to highest. $B$, An item analysis revealed that activity in the left angular gyrus correlated with the combinatorial strength of the word pairs. This correlation was performed using only the meaningful combinatorial pairs, and fMRI activity was extracted from an anatomically defined region of interest in the left angular gyrus (see Materials and Methods for details).

\section{Functional neuroimaging in healthy adults}

To examine the neural regions that support semantic combinations, we performed a whole-brain analysis of the fMRI data. We first identified regions where there was more activity for the more meaningful compared with the less meaningful combinations. This analysis revealed a significant cluster of activity in the left angular gyrus, shown in Figure $1 B$. No other brain regions showed significant activation for this contrast. We next determined whether this effect reflected a multimodal combinatorial process or one that was specific to a particular sensorimotor modality of semantic information. To address this question, we examined whether there were differential effects across the four sensorimotor categories of word pairs. As shown in Figure $1 C$, a repeated-measures ANOVA showed no differences between the sensorimotor word categories within the left angular gyrus $\left(F_{(3,54)}=0.13, p=0.94\right)$.

We next examined a continuous measure of conceptual combination within the more meaningful combinations and related this to a graded effect in the fMRI data by performing an item analysis. Figure $2 A$ shows the distribution of log co-occurrence frequencies across the meaningful combinations. As noted in Materials and Methods, this measure highly correlated with a subjective measure of combinatorial plausibility. We predicted that combinatorial strength would be related to the degree of activation in the angular gyrus. As shown in Figure $2 B$, we found that activity in the left angular gyrus was positively correlated with the degree of combinatorial strength across all items (Spear- man's $\rho=0.21, p<0.05$ Bonferroni corrected for the two ROIs tested). Within the ROI in the left anterior temporal lobe we found no correlation between neural activity and combinatorial strength (Spearman's $\rho=0.05, p>0.9$ Bonferroni corrected). Finally, we performed a whole-brain regression analysis of these data to determine whether other regions outside our preselected ROIs showed this effect. In this whole-brain regression, the right angular gyrus was the only region in which item-level neural activity correlated with combinatorial strength (a cluster of 5624 $\mu \mathrm{l}$ with a peak at [62-3838]).

We next examined whether left and right angular gyri were performing functionally distinct combinatorial operations by directly comparing the correlations using anatomically defined right and left angular gyrus ROIs. We performed a Fisher's $r$-to- $z$ transformation on the correlations between neural activity and combinatorial strength within each ROI and compared these. There was no significant difference between the effects in the left and right angular gyri $(z=0.3, p=0.76)$. Rather, the effects in the left and right angular gyri were highly correlated across word pairs (Spearman's $\rho=0.79, p<0.001$ ).

Together, these results demonstrate a relationship between neural activity in the angular gyrus and conceptual combination. These findings show that the angular gyrus is activated during the processing of meaningful combinations and that the degree of activation in the angular gyrus scales with a continuous measure of combinatorial strength. 


\section{Structural neuroimaging in healthy adults}

As discussed above, subjects displayed a processing advantage in their behavior for high combinatorial-strength word pairs relative to low combinatorialstrength word pairs (i.e., faster reaction times for items with high-combinatorial strength). However, there were individual differences in the extent to which subjects showed this processing advantage, shown in Figure $3 A$. Some subjects had a stronger processing advantage (in blue) while other subjects showed a weaker processing advantage (in red). We hypothesized that individual differences in the degree of this processing advantage would be related to individual differences in the structure of the left and right angular gyri.

We tested this hypothesis by examining cortical thickness within functionally defined regions of the left and right angular gyri. We also examined an anatomical region of interest in the left anterior temporal lobe. Indeed, we found that cortical thickness in the right angular gyrus was strongly correlated with the degree of the processing advantage for high relative to lowcombinatorial items (Fig. 3B; Spearman's $\rho=0.583, p=0.008$; all structural correlations were one-tailed and Bonferroni corrected for three multiple comparisons), whereas cortical thickness in the left angular gyrus and the left anterior temporal lobe showed no significant relationship with the combinatorial processing advantage (left angular gyrus: Spearman's $\rho=0.340, p=$ 0.183; left anterior temporal lobe: Spearman's $\rho=0.355, p=$ $0.158)$. This finding demonstrates that even in healthy adults, individual variability in the structure of the right angular gyrus is related to individual variability in the processing of combined semantic information.

One subject in the structural analysis was an outlier, with a cortical thickness value in the right angular gyrus $(3.2 \mathrm{~mm})$ that was $>3$ SDs away from the mean of the other subjects (mean 2.4 $\mathrm{mm}, \mathrm{SD}=0.26)$. We therefore removed this subject from the correlation analyses. We note, however, that even if this participant is left in the analysis, it does not change the significance of the correlations (right angular gyrus: $\rho=0.576, p=0.008$; left angular gyrus: $\rho=0.303, p=0.256$; left anterior temporal lobe: $\rho=0.354, p=$ $0.159)$.

\section{Structural neuroimaging in patients}

We next addressed the critical question of whether atrophy of the angular gyrus results in impaired comprehension of conceptual combinations by testing a group of patients with cortical atrophy from neurodegenerative disease (see Table 1, Fig. 4). We administered the same combinatorial task performed by healthy adults in the fMRI experiment. We additionally administered a separate single-word lexical decision task to assess the patients' singleword knowledge. We predicted that atrophy of the angular gyrus would result in a relatively greater impairment on combined concepts relative to impairments on single words. To test this, we calculated a difference score between performance on the combinatorial task and performance on the single-word task within

\section{B Cortical thickness in the angular gyrus correlates with combinatorial strength advantage}

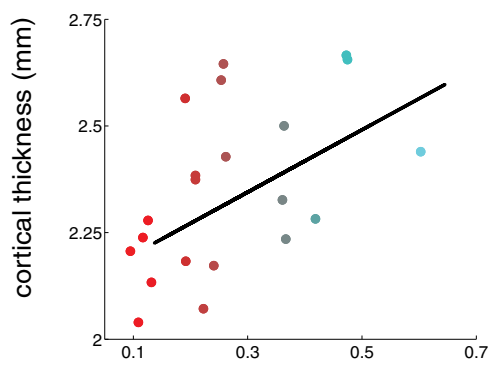

combinatorial strength advantage (rho)

Figure 3. In healthy adults, individual differences in performance on the combinatorial task were related to individual differantage were correlated with individual differences in the cortical thickness of the right angular gyrus (whole-brain between reaction time and the combinatorial strength of the stimuli within each subject, as shown in $\boldsymbol{A}$. They are calculated by taking the negative of the Spearman's $\rho$ values from a correlation of reaction time and combinatorial strength. See Results for an analysis of a single outlier in cortical thickness.

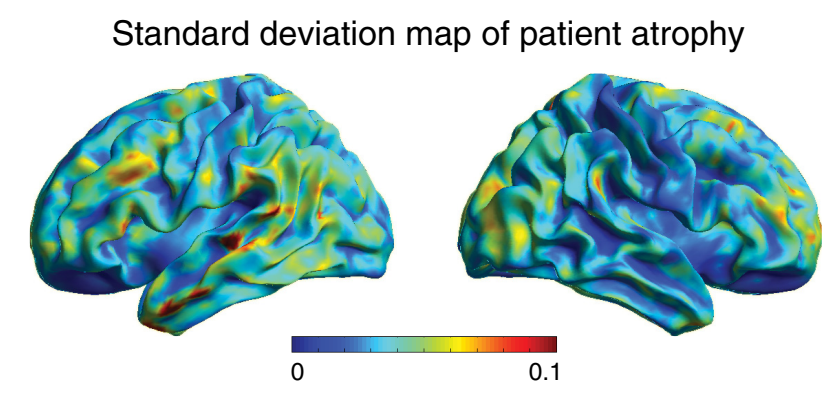

Figure 4. Whole-brain map of the SD of patient atrophy. The value in each voxel represents 1 SD of gray matter density across the 20 patients, where warmer colors indicate greater variability in atrophy.

each patient. This difference score provided a specific measure of the patients' relative performance on combined concepts, while controlling for general impairments with lexical access and other lower-level cognitive functions engaged by the single-word task.

We first examined whether the degree of impaired performance on combined concepts was related to atrophy in the region of the left angular gyrus that was activated in the fMRI study of healthy adults (i.e., Fig. $1 B$ ). Indeed, we found that in patients the degree of atrophy in this region was associated with the degree of impaired performance on the combinatorial task relative to the single-word task (Fig. 5A; Spearman's $\rho=-0.73, p<$ 0.001 , one tailed). We next performed a whole-brain regression analysis (Table 2), which revealed that the relative deficit on the combinatorial task was strongly associated with atrophy in the left and right angular gyri, shown in Figure $5 B$. There was considerable overlap of the anatomic findings from the patient experiment and the fMRI findings in healthy adults, as illustrated in Figure $5 C$. 
A Angular gyrus region of interest in patients

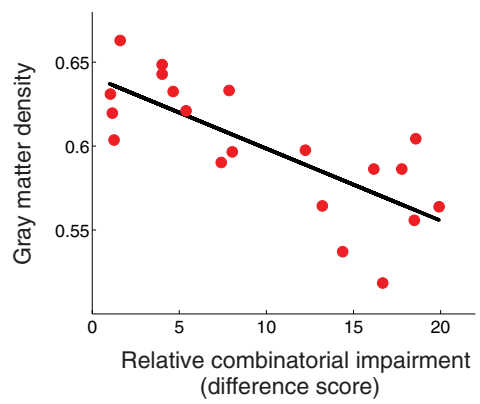

B Whole-brain regression of difference score in patients

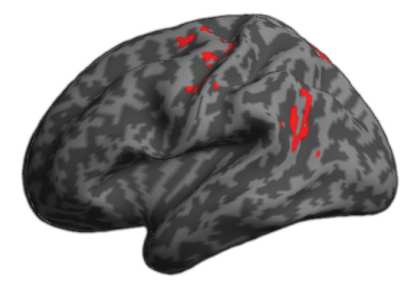

C Overlap of $\mathrm{fMRI}$ results in healthy subjects and patient results

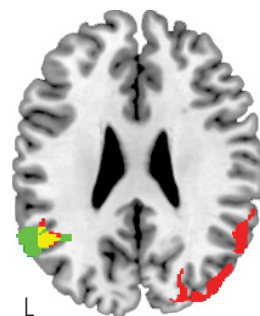

$Z=26$

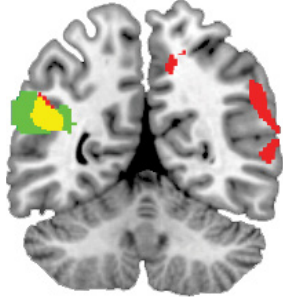

$Y=-55$

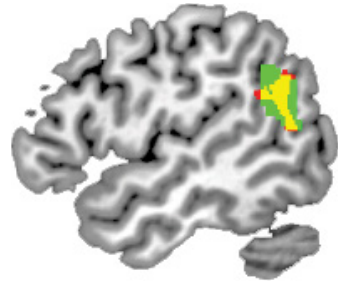

$X=-52$
fMRI results

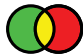

Figure 5. In patients with neurodegenerative disease, the degree of atrophy in the angular gyrus was related to the degree of specific impairment on combinatorial performance. $\boldsymbol{A}$, Gray matter density in the left angular gyrus of patients strongly correlated with the degree of impaired performance on the combinatorial task relative to the single-word task (relative combinatorial impairment score $=$ overall accuracy on the single-word task - overall accuracy on the combinatorial task). $\boldsymbol{B}, A$ whole-brain regression shows regions where gray matter atrophy was strongly related to the relative combinatorial impairment in patients. There was a strong effect in the angular gyrus. C, Overlap (shown in yellow) of the fMRI results from Figure $1 A$ (shown in green) and the patient regression results (shown in red). This illustrates the considerable overlap of the experimental findings from the fMRI and patient studies.

\section{Discussion}

An unresolved question in human cognition is how the brain integrates conceptual information into coherent representations. In this series of experiments we found converging evidence from patients and healthy adults that the angular gyrus is a critical region underlying this process. In healthy subjects, we found that activity in the angular gyrus is modulated by the integration of words into meaningful combinations. Furthermore, we found that the processing of combined concepts in healthy adults is related to individual differences in the structure of the angular gyrus. Finally, we found that atrophy of the angular gyrus in patients with neurodegenerative disease is associated with a greater impairment on combined concepts relative to individual concepts, demonstrating a necessary role for this region in conceptual combination. These novel findings provide important new insights into the neuroanatomic basis of conceptual combination, and suggest that a key function of the angular gyrus is to support the representation of integrated semantic information.

Our results are consistent with the known anatomic and functional properties of the angular gyrus. The cytoarchitecture and white matter connectivity of the angular gyrus are well suited to perform the heteromodal integrative functions required for a process like conceptual combination (Elston et al., 2001; Jacobs et al., 2001; Orban et al., 2004; Sherwood et al., 2008; Collins et al., 2010; Hill et al., 2010; Glasser and Van Essen, 2011). Additionally,
Patient results

comparative anatomic studies suggest that it has undergone a prominent evolutionary expansion in humans relative to monkeys (Orban et al., 2004; Sherwood et al., 2008; Hill et al., 2010). Functionally, it is one of the most commonly activated regions in studies of lexical-semantic memory (Binder et al., 2009), and it has been specifically implicated in the processing of sentences compared with word lists (Friederici et al., 2000; Vandenberghe et al., 2002; Humphries et al., 2006), with an activation profile that specifically correlates with the number of sequentially coherent words in a sentence (Pallier et al., 2011). Altogether, these neurobiological properties are consistent with a fundamental role for the angular gyrus in performing integrative functions in semantic memory.

Of the few studies that have specifically examined the neural correlates of basic combinatorial processing, some have emphasized the role of the anterior temporal lobe (Baron et al., 2010; Bemis and Pylkkänen, 2011, 2013) while other work has implicated the right angular gyrus (Graves et al., 2010) and left angular gyrus in this process (Bemis and Pylkkänen, 2013). Here, we found strong, converging evidence from three different experiments that the left angular gyrus is critical for the process of conceptual combination. We did not find any significant results that implicated the anterior temporal lobe in our comparisons. Although fMRI is known to be susceptible to signal loss in the medial and inferior temporal regions (Devlin et al., 2000), we also did not find evidence linking the anterior temporal lobe to combinatorial processing in our structural imaging studies, which are less susceptible to signal dropout in this region. It will be of interest in future work to determine the differential contribution of the anterior temporal lobe and the angular gyrus in integrative semantic processes.

The grammatical categories of the stimuli may also be relevant when interpreting differences across studies. For example, when reading adjective-noun pairs, one has a strong expectation that the adjective will be followed by a noun with which it should be combined. On the other hand, when reading noun-noun pairs, as used in Graves et al. (2010), readers may not expect the first noun to act as a modifier in a semantic combination. This may result in more semantic searching or re-evaluation when processing noun-noun combinations.

Past work has been limited to the use of categorical contrasts to identify the loci of anatomic regions that contribute to combinatorial processing. Here we build on previous findings and advance a novel framework for conceptual combination by characterizing a continuous metric that is critical for understanding combinatorial processes. We found that a metric of cooccurrence frequency strongly correlates with how plausible a combination is perceived to be and that this metric is associated with a graded neural response in the fMRI data as well as individual differences in how healthy adults process combined 
Table 2. MRI clusters from patient regression analysis

\begin{tabular}{|c|c|c|c|c|}
\hline$x$ & $Y$ & $Z$ & Cluster size $(\mu l)$ & Z-score \\
\hline 30 & -41 & -6 & 3694 & 5.00 \\
\hline 21 & -48 & -6 & & 4.09 \\
\hline 37 & -33 & -7 & & 3.69 \\
\hline 46 & -78 & 21 & 16326 & 4.70 \\
\hline 55 & -37 & 10 & & 4.48 \\
\hline 38 & -67 & 44 & & 4.48 \\
\hline-42 & -56 & 22 & 2301 & 4.64 \\
\hline-56 & -61 & 14 & & 4.14 \\
\hline-53 & -51 & 24 & & 3.37 \\
\hline 23 & -18 & 67 & 897 & 4.59 \\
\hline 18 & -23 & 71 & & 4.06 \\
\hline 32 & -18 & 58 & & 3.92 \\
\hline 33 & 0 & 56 & 532 & 4.50 \\
\hline 41 & 6 & 55 & & 3.97 \\
\hline-19 & -18 & 65 & 760 & 4.42 \\
\hline-24 & -7 & 55 & & 3.75 \\
\hline-15 & -14 & 72 & & 3.25 \\
\hline 24 & -73 & -10 & 1112 & 4.39 \\
\hline 11 & -76 & -4 & & 3.95 \\
\hline-41 & -16 & 53 & 2030 & 4.36 \\
\hline-30 & -32 & 52 & & 3.86 \\
\hline-43 & -14 & 38 & & 3.73 \\
\hline-22 & -76 & -5 & 1170 & 4.12 \\
\hline-21 & -57 & -7 & & 3.93 \\
\hline 31 & -24 & 52 & 1689 & 4.05 \\
\hline 47 & -7 & 53 & & 4.03 \\
\hline 34 & -15 & 46 & & 3.86 \\
\hline 58 & -26 & 18 & 763 & 4.05 \\
\hline 43 & -28 & 13 & & 3.43 \\
\hline
\end{tabular}

Significant clusters from the whole-brain regression analysis in patients with peaks and subpeaks. Coordinates are in MNI space.

concepts. By taking this continuous and objective measure into consideration, we were able test more fine-grained hypotheses about the neural basis of conceptual combination. Future studies may benefit from considering differences in co-occurrence frequency between more and less meaningful combinatorial conditions.

Our patient study is the first test of whether the angular gyrus is necessary for conceptual combination. Previous neuroanatomic studies of conceptual combination have been limited to analyses of functional activity. Here we examined the effects of cortical atrophy on conceptual combination and found that angular gyrus atrophy results in impaired comprehension of combined concepts relative to single-word concepts. However, it can be argued that processing two words is more challenging than processing single words, and thus our difference score might reflect general task difficulty. However, if the contribution of the angular gyrus reflects general task demands, one would expect the low-combinatorial items in our fMRI study to elicit the strongest activation, which is the opposite of the observed fMRI effect. Altogether, the most consistent account for our findings is that the angular gyrus supports the comprehension of combined concepts.

Research on conceptual combination has been framed in a number of different ways in the literature. One line of investigation has focused on the processing of semantically ambiguous or anomalous phrases (also referred to as semantic integration). For example, the N400 effect in electrophysiological studies is strongly elicited by words that are unexpected given the preceding context (e.g., "I like my coffee with cream and socks"; Kutas and Hillyard, 1980; Kutas and Federmeier,
2000). These effects are often thought to reflect a mechanism for integrating the meaning of a word with the preceding semantic context, where more unification is elicited by increasingly unexpected words (Hagoort, 2005). An alternative interpretation has been that lexical access to the target word is made more challenging by the incongruent context and thus increased effort is required (Lau et al., 2008). Our experiments and recent studies from other groups have begun to examine the mechanisms involved in fluent conceptual combination in language, in which basic conceptual constituents are integrated coherently into higher-level representations (Graves et al., 2010; Bemis and Pylkkänen, 2011). Although each of these approaches focuses on a different cognitive process, they are all relevant to understanding the full spectrum of conceptual combination. We suggest that the differences in current theoretical frameworks partly reflect the lack of an established taxonomy for the cognitive processes in conceptual combination, rather than fundamental differences in what constitutes conceptual combination.

In this series of studies we analyzed conceptual combinations via a lexical modality of input. However, considering these findings in light of other work it seems likely that the semantic information in the angular gyrus is independent of the material of input (e.g., lexical, pictorial). Indeed, a recent study by Fairhall and Caramazza (2013) showed that the angular gyrus is one region that can successfully cross-classify between visual objects and their corresponding single-word representations (e.g., between the word "apple" and a picture of an apple). Future work is needed to explicitly characterize whether the combinatorial mechanisms in this region are independent of the lexical and pictorial material used to access conceptual information.

Across many studies of lexical semantics the angular gyrus has been implicated bilaterally in lexical-semantic processing (Binder et al., 2009; Bonner et al., 2013). Our results are also consistent with a bilateral contribution, although there may be subtle hemispheric differences. Our fMRI results demonstrate that combinatorial processing modulates activity in both the left and right angular gyri. Indeed, activity in the left and right angular gyri did not differ and were highly correlated in our item analysis. Furthermore, in the patient analysis the degree of atrophy in both the left and right angular gyri correlated with impaired combinatorial processing. However, when we examined individual differences in combinatorial processing across healthy adults within the structural MRI analysis, we found that the strongest structure-function relationship was in the right angular gyrus, with no indication of a similar relationship in the left hemisphere. With typical left-hemisphere language dominance in righthanders, lexical-semantic processing may rely more consistently on the left angular gyrus across subjects for all types of combinatorial stimuli. However for some participants, an advantage may be gained by additionally recruiting the right angular gyrus and thus, the right angular gyrus may be more sensitive to individual differences across subjects in lexical-semantic processing (Heim et al., 2010).

In conclusion, we have shown in a series of experiments that the neural mechanisms for conceptual combination rely on the heteromodal association cortex of the angular gyrus. We found that activity in the angular gyrus is modulated by conceptual combination in healthy adults, and that combinatorial performance is sensitive to the degree of angular gyrus atrophy in patients. These converging findings indicate that 
the angular gyrus plays a critical role in integrating semantic information.

\section{References}

Albert MS, DeKosky ST, Dickson D, Dubois B, Feldman HH, Fox NC, Gamst A, Holtzman DM, Jagust WJ, Petersen RC, Snyder PJ, Carrillo MC, Thies B, Phelps CH (2011) The diagnosis of mild cognitive impairment due to Alzheimer's disease: recommendations from the National Institute on Aging-Alzheimer's Association workgroups on diagnostic guidelines for Alzheimer's disease. Alzheimers Dement 7:270-279. CrossRef Medline

Avants BB, Anderson CL, Grossman M, Gee JC (2008) Symmetric normalization for patient-specific tracking of longitudinal change in frontotemporal dementia. Med Image Anal 12:26-41. CrossRef Medline

Baron SG, Thompson-Schill SL, Weber M, Osherson D (2010) An early stage of conceptual combination: superimposition of constituent concepts in left anterolateral temporal lobe. Cogn Neurosci 1:44-51. CrossRef Medline

Bedny M, Aguirre GK, Thompson-Schill SL (2007) Item analysis in functional magnetic resonance imaging. Neuroimage 35:1093-1102. CrossRef Medline

Bemis DK, Pylkkänen L (2011) Simple composition: a magnetoencephalography investigation into the comprehension of minimal linguistic phrases. J Neurosci 31:2801-2814. CrossRef Medline

Bemis DK, Pylkkänen L (2013) Basic linguistic composition recruits the left anterior temporal lobe and left angular gyrus during both listening and reading. Cereb Cortex 23:1859-1873. CrossRef Medline

Binder JR, Desai RH, Graves WW, Conant LL (2009) Where is the semantic system? A critical review and meta-analysis of 120 functional neuroimaging studies. Cereb Cortex 19:2767-2796. CrossRef Medline

Bonner MF, Grossman M (2012) Gray matter density of auditory association cortex relates to knowledge of sound concepts in primary progressive aphasia. J Neurosci 32:7986-7991. CrossRef Medline

Bonner MF, Peelle JE, Cook PA, Grossman M (2013) Heteromodal conceptual processing in the angular gyrus. Neuroimage 71:175-186. CrossRef Medline

Brysbaert M, New B (2009) Moving beyond Kucera and Francis: a critical evaluation of current word frequency norms and the introduction of a new and improved word frequency measure for American English. Behav Res Methods 41:977-990. CrossRef Medline

Collins CE, Airey DC, Young NA, Leitch DB, Kaas JH (2010) Neuron densities vary across and within cortical areas in primates. Proc Natl Acad Sci U S A 107:15927-15932. CrossRef Medline

Das SR, Avants BB, Grossman M, Gee JC (2009) Registration based cortical thickness measurement. Neuroimage 45:867-879. CrossRef Medline

Devlin JT, Russell RP, Davis MH, Price CJ, Wilson J, Moss HE, Matthews PM, Tyler LK (2000) Susceptibility-induced loss of signal: comparing PET and fMRI on a semantic task. Neuroimage 11:589-600. CrossRef Medline

Elston GN, Benavides-Piccione R, DeFelipe J (2001) The pyramidal cell in cognition: a comparative study in human and monkey. J Neurosci 21: RC163. Medline

Fairhall SL, Caramazza A (2013) Brain regions that represent amodal conceptual knowledge. J Neurosci 33:10552-10558. CrossRef Medline

Fodor JA, LePore E (2002) The composionality papers. New York: Oxford UP.

Friederici AD, Meyer M, von Cramon DY (2000) Auditory language comprehension: an event-related fMRI study on the processing of syntactic and lexical information. Brain Lang 74:289-300. CrossRef Medline

Glasser MF, Van Essen DC (2011) Mapping human cortical areas in vivo based on myelin content as revealed by T1- and T2-weighted MRI. J Neurosci 31:11597-11616. CrossRef Medline

Gorno-Tempini ML, Hillis AE, Weintraub S, Kertesz A, Mendez M, Cappa SF, Ogar JM, Rohrer JD, Black S, Boeve BF, Manes F, Dronkers NF, Vandenberghe R, Rascovsky K, Patterson K, Miller BL, Knopman DS, Hodges JR, Mesulam MM, Grossman M (2011) Classification of primary progressive aphasia and its variants. Neurology 76:1006-1014. CrossRef Medline

Graves WW, Binder JR, Desai RH, Conant LL, Seidenberg MS (2010) Neural correlates of implicit and explicit combinatorial semantic processing. Neuroimage 53:638-646. CrossRef Medline
Hagoort P (2005) On Broca, brain, and binding: a new framework. Trends Cogn Sci 9:416-423. CrossRef Medline

Hayasaka S, Phan KL, Liberzon I, Worsley KJ, Nichols TE (2004) Nonstationary cluster-size inference with random field and permutation methods. Neuroimage 22:676-687. CrossRef Medline

Heim S, Grande M, Meffert E, Eickhoff SB, Schreiber H, Kukolja J, Shah NJ, Huber W, Amunts K (2010) Cognitive levels of performance account for hemispheric lateralisation effects in dyslexic and normally reading children. Neuroimage 53:1346-1358. CrossRef Medline

Hill J, Inder T, Neil J, Dierker D, Harwell J, Van Essen D (2010) Similar patterns of cortical expansion during human development and evolution. Proc Natl Acad Sci U S A 107:13135-13140. CrossRef Medline

Hume D (1739/1978) A treatise of human nature (Selby-Bigge LA, Nidditch $\mathrm{PH}$, eds.). Oxford: Clarendon.

Humphries C, Binder JR, Medler DA, Liebenthal E (2006) Syntactic and semantic modulation of neural activity during auditory sentence comprehension. J Cogn Neurosci 18:665-679. CrossRef Medline

Jacobs B, Schall M, Prather M, Kapler E, Driscoll L, Baca S, Jacobs J, Ford K, Wainwright M, Treml M (2001) Regional dendritic and spine variation in human cerebral cortex: a quantitative Golgi study. Cereb Cortex 11: 558-571. CrossRef Medline

Kutas M, Federmeier KD (2000) Electrophysiology reveals semantic memory use in language comprehension. Trends Cogn Sci 4:463-470. CrossRef Medline

Kutas M, Hillyard SA (1980) Reading senseless sentences: brain potentials reflect semantic incongruity. Science 207:203-205. CrossRef Medline

Lau EF, Phillips C, Poeppel D (2008) A cortical network for semantics: (de)constructing the N400. Nat Rev Neurosci 9:920-933. CrossRef Medline

Maldjian JA, Laurienti PJ, Kraft RA, Burdette JH (2003) An automated method for neuroanatomic and cytoarchitectonic atlas-based interrogation of fMRI data sets. Neuroimage 19:1233-1239. CrossRef Medline

Martin A (2007) The representation of object concepts in the brain. Annu Rev Psychol 58:25-45. CrossRef Medline

McKhann GM, Knopman DS, Chertkow H, Hyman BT, Jack CR Jr, Kawas CH, Klunk WE, Koroshetz WJ, Manly JJ, Mayeux R, Mohs RC, Morris JC, Rossor MN, Scheltens P, Carrillo MC, Thies B, Weintraub S, Phelps CH (2011) The diagnosis of dementia due to Alzheimer's disease: recommendations from the National Institute on Aging-Alzheimer's Association workgroups on diagnostic guidelines for Alzheimer's disease. Alzheimers Dement 7:263-269. CrossRef Medline

Medler DA, Binder JR (2005) MCWord: an on-line orthographic database of the English language. http://www.neuro.mcw.edu/mcword/.

Mesulam MM, Mesulam MM (2000) Behavioral neuroanatomy: large-scale networks, association cortex, frontal syndromes, the limbic system, and hemispheric specializations. In: Principles of behavioral and cognitive neurology, pp 1-120. New York: Oxford UP.

Murphy G (2002) The big book of concepts. Cambridge, MA: MIT.

Orban GA, Van Essen D, Vanduffel W (2004) Comparative mapping of higher visual areas in monkeys and humans. Trends Cogn Sci 8:315-324. CrossRef Medline

Pallier C, Devauchelle AD, Dehaene S (2011) Cortical representation of the constituent structure of sentences. Proc Natl Acad Sci U S A 108:25222527. CrossRef Medline

Pandya DN, Seltzer B (1982) Association areas of the cerebral cortex. Trends Neurosci 5:386-390. CrossRef

Patterson K, Nestor PJ, Rogers TT (2007) Where do you know what you know? The representation of semantic knowledge in the human brain. Nat Rev Neurosci 8:976-987. CrossRef Medline

Pulvermüller F (2005) Brain mechanisms linking language and action. Nat Rev Neurosci 6:576-582. CrossRef Medline

Rascovsky K, Hodges JR, Knopman D, Mendez MF, Kramer JH, Neuhaus J, van Swieten JC, Seelaar H, Dopper EG, Onyike CU, Hillis AE, Josephs KA, Boeve BF, Kertesz A, Seeley WW, Rankin KP, Johnson JK, Gorno-Tempini ML, Rosen H, Prioleau-Latham CE, et al. (2011) Sensitivity of revised diagnostic criteria for the behavioural variant of frontotemporal dementia. Brain 134:2456-2477. CrossRef Medline

Seltzer B, Pandya DN (1978) Afferent cortical connections and architectonics of the superior temporal sulcus and surrounding cortex in the rhesus monkey. Brain Res 149:1-24. CrossRef Medline 
Sherwood CC, Subiaul F, Zawidzki TW (2008) A natural history of the human mind: tracing evolutionary changes in brain and cognition. J Anat 212:426-454. CrossRef Medline

Tustison NJ, Avants BB, Cook PA, Zheng Y, Egan A, Yushkevich PA, Gee JC (2010) N4ITK: improved N3 bias correction. IEEE Trans Med Imaging 29:1310-1320. CrossRef Medline

Tustison NJ, Cook PA, Klein A, Song G, Das SR, Duda JT, Kandel BM, van Strien N, Stone JR, Gee JC, Avants BB (2014) Large-scale evaluation of ANTs and FreeSurfer cortical thickness measurements. Neuroimage 99: 166-179. CrossRef Medline

Tzourio-Mazoyer N, Landeau B, Papathanassiou D, Crivello F, Etard O, Delcroix N, Mazoyer B, Joliot M (2002) Automated anatomical labeling of activations in SPM using a macroscopic anatomical parcellation of the MNI MRI single-subject brain. Neuroimage 15:273-289. CrossRef Medline

Vandenberghe R, Nobre AC, Price CJ (2002) The response of left temporal cortex to sentences. J Cogn Neurosci 14:550-560. CrossRef Medline

Worsley KJ, Evans AC, Marrett S, Neelin P (1992) A three-dimensional statistical analysis for $\mathrm{CBF}$ activation studies in human brain. J Cereb Blood Flow Metab 12:900-918. CrossRef Medline

Yeterian EH, Pandya DN (1985) Corticothalamic connections of the posterior parietal cortex in the rhesus monkey. J Comp Neurol 237:408-426. CrossRef Medline 